Moreover, with the decreasing length of stay in hospitals and the increasing outpatient delivery of complex medical and surgical care, we now need to look at the infrastructure and essential activities for infection control programs in out-of-hospital settings, such as longterm care, home care, and ambulatory surgery. Clearly, infection control programs will need to adapt to the differences between these venues and the traditional in-hospital setting. Similarly, the increasing application of epidemiology to the broader discipline of healthcare delivery warrants a blueprint for the expanded use of epidemiological principles and methods for prevention and control of noninfectious adverse events and as tools in quality assessment and outcome measurement. These two issues-infection control in the out-of-hospital setting and use of epidemiological principles for surveillance and control of noninfectious nosocomial events-should keep our next consensus panels flying.

\section{REFERENCES}

1. Homer CJ. Asthma disease management. $N$ Engl J Med 1997;337:1461-1463.

2. Scheckler WE, Brimhall D, Buck AS, Farr BM, Friedman C, Garibaldi RA, et al. Requirements for infrastructure and essential activities of infection control and epidemiology in hospitals: a consensus panel report. Infect Control Hosp Epidemiol $1998 ; 19: 114-124$

\title{
Update on Reuse of Hemodialyzers
}

\section{Gina Pugliese, RN, MS Martin S. Favero, PhD}

The Council on Dialysis of the National Kidney Foundation recently convened an expert panel to evaluate current practice related to the reuse of hemodialyzers and to review and evaluate literature related to reuse published since the last report of the National Kidney Foundation in 1988.

The foundation takes no position for or against dialyzer reuse. The principal reason for the practice of reuse is economical. In view of the uncertainties related to the safety and biological impact of reuse procedures, the task force recommended that a full discussion of the issue of reuse and its potential beneficial and detrimental effects be undertaken with each patient. The paper found no conclusive evidence that morbidity or mortality differed with single use or reuse. Microbial contamination of the water used for dialyzer reprocessing increases patient morbidity; the chemical quality of water used for dialyzer reprocessing should, at least, fall within the same standards as those recommended for product water intended for hemodialysis. Dialyzers should not be reprocessed from patients who have tested positive for hepatitis B surface antigen. The effects of reprocessing high-flux dialyzers on $\beta 2$-microglobulin clearance are dependent on the reprocessing technique, the number of reuses, and the nature of the dialyzer membrane used; there are insufficient data on the effects of reuse on $\beta 2$ microglobulin behavior to make uniform recommendations. Untoward effects of reused dialyzers still may occur in spite of rigorous adherence to Association for Advancement of Medical Instrumentation (AAMI) guidelines. For example, use of the total cell volume method for assessing changes in small molecule clearances will not show the loss of performance attributable to dialysate shunting. For this reason, the measurement of Kt/V for urea, as recommended by the AAMI, or the determination of the urea reduction ratio is recommended strongly at least monthly to gauge the adequacy of the dialysis procedure. Given the significant fall in dialyzer efficiency for urea removal that can occur after repeated uses of a dialyzer, dialysis prescriptions in units practicing reuse should be designed to deliver a Kt/V or urea reduction ratio value that exceeds the dose used for patients treated with single-use dialyzers to make allowance for any possible reuseinduced reduction in dialyzer efficiency. Technicians and other personnel responsible for the reprocessing of dialyzers should receive proper training and certification.

FROM: Task Force on Reuse of Dialyzers, Council on Dialysis, National Kidney Foundation. National Kidney Foundation report on dialyzer reuse. Am J Kidney Dis 1997;30:859-871. 